\title{
Artificial Intelligence-Virtual Trainer: Innovative Didactics Aimed at Personalized Training Needs
}

\author{
Zhisheng Chen ${ }^{1}$ (1)
}

Received: 24 October 2021 / Accepted: 1 February 2022

(C) The Author(s), under exclusive licence to Springer Science+Business Media, LLC, part of Springer Nature 2022

\begin{abstract}
First, the benefits of AI-based training for business development are presented. Then, the theoretical literature on various stages from traditional training to AI-based training is analyzed. Finally, we analyze AI applications in the training process and managerial response. This study presents a framework for the application of artificial intelligence (AI) technology to training and managerial challenges. AI tools can be applied to the training process, including knowledge management (KM), needs analysis, training organization, and results feedback. AI-based training transforms organizations into knowledge organizations that can meet the demands of personalized training and improve learning quality. AI tools bring about a shift in the training phases of knowledge base creation, needs surveys, the organization of training, and feedback on results.
\end{abstract}

Keywords Artificial intelligence · Virtual trainer: Innovative didactics · Personalized training needs

\section{Introduction}

While the quadruple helix encourages the knowledge society perspective, the quintuple helix emphasizes the necessary socio-ecological transformation, which is also the driving force for knowledge innovation (Carayannis et al., 2012). The quadruple and

\footnotetext{
Zhisheng Chen received a master's degree in Business Administration from Nanjing University of China and is currently studying for a PhD in Management Science and Engineering at Nanjing University of Aeronautics and Astronautics. He has worked in the areas of human resource management, business management consulting, and macro strategy research.
}

This article is part of the Topical Collection on AI in the Knowledge Economy and Society: Implications for Theory, Policy and Practice

Zhisheng Chen

njuczs@nuaa.edu.cn; 776271378@qq.com

1 College of Economics and Management, Nanjing University of Aeronautics and Astronautics, Jiangning District, No.29, General Avenue, Nanjing, China 
quintuple helices are grounded in democracy and ecology (Carayannis et al., 2021). Knowledge innovation and learning undoubtedly appear to be important for employees. With the need to continually upskill employees in organizations required by the rapid innovation and disruption of today's business processes and technologies, training demands are becoming more personalized and adaptable to changing practices. The core of training and development efforts is to help employees learn to improve their knowledge, skills, and competencies for current and future work. Trainees are also expected to receive personalized training in relevant areas of expertise and operational skills that will help them to overcome career bottlenecks (LiT et al., 2019).

However, traditional training is faced with a few problems, such as a single approach to the training, training content that does not effectively meet the needs of employees, training objectives that are not integrated with organizational objectives, and training that lacks systematicity. These have led to a failure to meet the needs of the organization and the trainees (LiT et al., 2019).

The shortcomings of most traditional training methods and techniques usually produce "trained novices" at most (Ong \& Ramachandran, 2003). In other words, they orient trainees to principles and concepts, pose them with relatively easy questions to try out this acquired knowledge, and then provide them with some opportunities to use it in exercises or situations. However, this requires broad exercises in solving complicated issues in a variety of contexts, coupled with personalized tutoring and feedback to become a truly skilled employee. The cost limits the amount of conventional training that can be provided and employees must hone their skills on the job. It is also very expensive to put novices with average conventional training on the job because of the additional costs associated with sub-optimal performance.

With the development of information technology and artificial intelligence, there is scope for further exploration of ways to address these issues. An ever-growing range of enterprises is introducing technologies-based training to enhance or replace traditional training forms (Salas et al., 2012).

Computer-based multimedia training (CBT) technology and web-based training (WBT) technology alleviate the problems of traditional training, but CBT systems do not provide intelligent, personalized training, evaluation, and feedback that students require to gain extensive and in-depth professional knowledge. While WBT alleviates the difficult logistics associated with delivering and setting up study software, the didactic limitations are equally evident. While accessing the Internet, learners often depend on asynchronous exchanges to obtain assistance from tutors and to solve their difficulties. When problems are encountered, they must await the assistance of online tutors. Based on the tutor-to-student rate, it can take a while for the tutor to help the student. The cost of assigning a tutor to each student is too high. The challenge for CBT and WBT technologies is therefore to encode the subject knowledge and teaching skills of a company's best employees into software that will allow them to enjoy the benefits of training (Ong \& Ramachandran, 2003).

Ong and Ramachandran (2003) suggest that training performance and return on investment (ROI) can be improved with AI technology, which can achieve the goal of cost-effective scenarios or exercises. He noted that if trainees receive AI intelligence training, they will perform two standard deviations better than in traditional 
teaching methods. Unlike previous training, the focus of AI-based training has shifted from instructor-centric to learner-centric.

Artificial intelligence should be applied to enterprise training to take on and help enterprises solve their training challenges. When recruiting and hiring new employees, the main challenge is to quickly and effectively make them thoroughly aware of and understand the organization's internal policies and procedures. Machine learning features are being incorporated into various HR software systems to facilitate more effective onboarding (Iqbal, 2018). Even more comprehensively, the use of various AI technologies can help enterprises develop a learning organizational culture, avoiding the common instructional design model based on traditional competency model analysis. What can be found, AI-based training advantages are manifested in three areas.

1. AI may help drive the fundamental principles of KM. It can convert personal knowledge into group one. Knowledge repositories are developed in the KM system to normally record the knowledge in an online manner (Liebowitz, 2001).

2. Enterprises may leverage AI technology, applying the key algorithm and the content database to enable effective and rapid learning experiences. Artificial intelligence will not only enhance learning efficiency and quality but also greatly decrease the management and operation of offline and online training. AI-based training systems would redefine the basic logic of training design. To achieve the core task of transferring knowledge from defining learning methods to defining problem-solving, the construction of "wisdom" will become a core component of curriculum designers in the AI era.

3. In addition, the AI can recommend training programs and external activities to better manage staff holistically, freeing up a lot of time for training practitioners to focus on other priorities (Iqbal, 2018). It supplies analytical support, freeing human trainers from mechanical jobs to perform more valuable tasks (Luckin \& Cukurova, 2019).

In any case, this new trend of making HR practices dependent on AI tools has the potential to deliver significant benefits in terms of increased employee motivation and workplace productivity. Today, AI is already beginning to have a huge impact on the way companies train and develop their employees (Iqbal, 2018). It is expected that the digital transformation of training across the enterprise will continue to grow rapidly after the global victory over the COVID-19 crisis. The prospect of training that enables AI tools has arrived (Iqbal, 2018).

Some theoretical studies and training practices have concentrated on traditional teaching or mobile e-learning. With the development of digital technology, especially artificial intelligence, and the rise in demand for personalized training, past training methods are no longer able to meet personalized needs. The adoption of AI-based training can effectively make up for the lack of personalized training. AI tools can provide personalized training through the intervention of the training process, thus improving the training effect. 
This is a qualitative study and attempts to explain how AI is beneficially integrated and effective for training. The study presents a framework for AI-based training processes and corresponding management challenges, which reflects the main components of the study. AI application to training processes involves knowledge base building, training needs mining, organizing training, and results feedback.

1. Knowledge base building: AI technology can transform corporate knowledge into group knowledge and build a knowledge base. In order to express knowledge in the knowledge base, knowledge categories and diagrams are usually built up.

2. Training demand mining: According to the job requirements, AI technology can form customized employee courses for employees who learn from the huge knowledge base through big data analysis. Artificial intelligence can systematically analyze the starting skills, cognitive level, job requirements, and other elements of employees, and continuously learn from their behavioral data to refine employee portraits.

3. Organizational training: It mainly performs in terms of providing personalized trainers, meeting personalized training needs, adopting micro-dose of learning, observing learning status, robot Q\&A, and scenario simulation.

4. Feedback on training results: AI can help trainees automatically record training data, evaluate each individual's performance, and provide them with useful comments and feedback during the training process.

However, AI-based training also brings corresponding management challenges, mainly in terms of cost, data security and legal risks, job replacement, equity, negative attitudes, and training outcome measurement.

1. Cost: Developing good AI tools requires good algorithms, and the corresponding cost is expensive. Companies can gradually build AI-based training transformation programs through internal data analysis systems.

2. Data security and legal risks: AI-based training needs to collect external and internal data, which can lead to data leakage risks. Data security and privacy tools need to be established.

3. Job replacement and equity: There are concerns that AI involvement in training will lead to the job loss of human trainers; in fact, the emergence of AI will reduce people's repetitive tasks and increase the strategic roles and tasks of human trainers.

4. Negative attitudes: People worry that communication with machines lacks human emotions and organizations can shape an inclusive corporate culture to receive the innovative idea.

5. Measurement of outcomes: It is difficult to measure training outcomes irregularly, and organizations are advised to adopt existing measurements and improve training activities through recursive analysis. 
The objective of the research is to explore the process of AI tools involved in the training process. The study found that AI-based training can change the traditional training approach to meet individualized training needs. While discussing its benefits, this study also highlights the challenges that AI-based training presents.

\section{Literature}

\section{Al Definition, Technology Classification}

Artificial intelligence is defined as "the study of how to make computers do things that people are better at" (Poll et al., 2021). In mimicking the information processes of humankind's thought and consciousness, it may rapidly search databases, extract information, efficiently answer our questions, and give the best answers in a straightforward and rational manner. It is "the skill of a system to correctly explain external datum, learn valuable competencies from that datum, and apply those learnings to finish specific tasks and goals by flexible adaptation" (Haenlein \& Kaplan, 2019).

The AI technology may be analyzed from three levels: the foundational support layer, the platform framework layer, and the domain technology layer (Jia et al., 2018).

1. Foundational support layer. The major elements for the success of AI technology in the layer are big data, computing power, and new models. Big data powers the development of artificial intelligence, while enhanced algorithmic models greatly improve machine learning capabilities (Jia et al., 2018).

2. Framework layer. This layer focuses on designing deep learning frameworks. It is expected that advanced deep learning techniques will be applied with little deployment and customization, validly decreasing the workload on enterprises and developers (Jia et al., 2018).

3. Domain technology layer. This includes computer vision technologies, sentiment analysis, and natural language processing technologies (Jia et al., 2018).

\section{Evolution in Al from the Traditional Age to the New Age}

\section{Before the Second World War}

The origins of training and development can be traced back to the first introduction of the concept of apprenticeship in the Middle Ages. The late eighteenth century witnessed the industrial revolution, which resulted in the need for highly skilled workers to run mass production effectively in a few countries. The early nineteenth century witnessed the origins of vocational schools in order to develop skills for specific trades (Werner \& DeSimone, 2011). Later, in the twentieth century, the First World War shortened the existing apprenticeship programs. At that time, there was a need to develop industrial operating skills in a shorter period and within a wider social context. Over time, vocational instructional training became the mainstream workforce in the training field (Werner \& DeSimone, 2011). 


\section{Post-World War II-the 1980s}

After World War II, training and development practices began to emerge worldwide. These practices were inspired by the launch of the Training Within Industry (TWI) service by the USA during the Second World War (Maity, 2019).

As the peacetime economy took shape, a new profession-training-began to enter several industries. The inspiration for this came from these training directors, who later led workforces cross-organizational development programs (Torraco, 2016). 1984 saw the development of the Instructional System Development (ISD) model (Reiser, 2001), which for the first time referred to the requirement for a training needs assessment outline and its content prior to the design of training courses. Training needs to be shifted to such a process in that the pre-training analysis process required less time (Rossett, 2009), and one that could integrate learning with the work of the employee (Ellinger \& Cseh, 2007).

\section{The 1990s-Now}

This was a time when the focus of both research and organizations shifted to enhancing employee involvement in development and learning activities. Employees' perceptions of the work environment and environmental factors prior to training all contribute to individual differences in learning (Bell et al., 2017). Researchers and organizations have also begun to place learners in different training programs and models. Many of them have studied how different programs like mastery training and mistake training can affect self-regulation procedures and how these programs affect individual differences and thus training outcomes (Bell et al., 2017).

An approach to measuring the impact of training programs on the enterprises extended their focus from the individual to the team and the organization (Aguinis \& Kraiger, 2009). Because team members became more mobile, employees found themselves working in multiple teams at the same time (Ellis et al., 2005). Communication and collaboration technologies have supported the effectiveness of various team training methods (Salas et al., 2008). The Internet is used to deliver educational programs and to rapidly deliver content to a large number of learners (Raab et al., 2001). Some organizations start to implement online training programs and drive research into how to make these programs more engaging and effective for their employees.

A mobile technology-mediated approach can increase engagement and confidence in learning (Bray \& Tangney, 2016). Internet-based teaching procedures or imbedded job-related software are becoming more prevalent in the industry (Aguinis \& Kraiger, 2009). E-learning in organizations has been largely promoted in order to meet the development of the advanced innovation, the digital media, and useful interactive instruments (Noe et al., 2014). 


\section{Transformation of Al-Based Training from Contemporary to Future}

While the use of technology in organizations to deliver training is increasing, research on e-learning in labor enterprises has declined significantly after 2016 (Kaizer et al., 2020). There is a shift in training needs that can identify future trends and development practices for AI in training. The world seems to be currently challenged by a race in knowledge innovation and production between developing emerging autocracies and democracies. In the further co-evolution of knowledge economy, knowledge society, and knowledge democracy, organizations have to seize the opportunity of creative design (Carayannis \& Campbell, 2014).

For individuals who wish to observe an intuitive e-learning interface that facilitates online and personalized learning, the future needs of training are to be met in the "SIP" model (Maity, 2019). The "SIP" model refers to an AI tool that allows for better seamless knowledge learning alignment, human-like conversational communication, and personalized training needs fulfillment. The benefits of adopting this model include easy access to learning models whenever and wherever employees need them, more intuitive and natural AI-based training programs through an intelligent user interface communication displays, and the ability to identify individual learning characteristics of trainees and reduce human bias.

1. Seamless. Knowledge management practices are already being implemented in some organizations, considering a large number of training materials and data sectors that have been accumulated in organizations. But what is needed is at the same time to make these data and learning modules easily accessible to employees where they need them. Organizations can leverage individual learning habits and preferences to design smarter platforms for delivering training content. AI allows for seamless knowledge learning interfacing (Zahra et al., 2014).

2. Intuition. Training practices move from the classroom to the mobile screen. Without the instructor, the model needs to be intuitive and adaptable to the user. For many organizations, the expectation is to make training programs more intuitive and closer to natural expectations. Artificial intelligence can play a key role in filling this gap. Adopting artificial intelligence for training, it can be in the form of human-like conversations and intelligent user interfaces for on-screen communication. The integration of artificial intelligence and virtual reality can allow people to experience live visual simulations of them completing work with a real-time training experience (Taylor, 2017).

3. Personalization. Without knowledge democracy, the further advancement of knowledge and innovation is severely constrained (Carayannis \& Campbell, 2021). People expect more from knowledge democracy. In the late nineteenth and early twentieth centuries, training plans were distributed to a group of professionals. But present needs have changed to the point where they are designed for each employee individually, considering learner characteristics. AI has the ability to recognize learning characteristics and to design training programs according to these characteristics. AI allows for less humankind bias and intervention in 
identifying training needs and explaining individual employee learning styles. Even when designing training programs, whether offline or online, it could be as intuitive and personalized as possible.

If all three features are present at the same time, the individualized training needs of future employees can be met. By adopting AI-based training, the requirements of SIP can be achieved. The training changes brought about by AI are therefore beneficial, both for the individual and the organization. AI technology should be a key area of investment for HR practitioners, as the technology has the potential to take HRM to new levels.

\section{Research Methodology}

This section is an extension of the systematic literature review of AI-based training and explores perspectives from mainstream research. The first step of the review was to search for keywords that were used to identify publications on AI-based training. This study collected and analyzed AI-based training literature with the help of commonly used academic websites such as Scopus, WOS, and Google Scholar Academic, using the keywords AI or artificial or robot or algometric or electric + train or learn or development or tutor. There are more than 3,600 recommended articles, but less than 21 articles are about AI-based training research.

This article also draws on AI-based training practices from technology companies such as IBM and Baidu as a supplement to this study. AI recruitment research has been ongoing for some time, and AI-based training has only developed in the last few years. The increasing trend of AI-based training applications in the future and the lack of literature research also give scholars more challenges and opportunities.

\section{Application of Al in the Training Process}

Bell et al. (2017) argue that the training planning includes the choice of instructional techniques and ways suitable to trainees, the definition of learning goals, and the selection and transfer of instructional learning support strategies. Abbad and Borges-Andrade (2004) suggest training subsystems including needs evaluation, instructional planning, and implementation. These sub-systems comprise a range of inter-dependent strategic actions that together constitute the technical and training system for teaching and development.

Based on the content of training planning and subsystems described by scholars, training supported by AI systems can be divided into four phases, such as building a knowledge base, training needs mining, organizing training, and feedback on training effectiveness. Throughout the training phase, it can be found that AI identifies training demands, maps the trainer, and uses relevant inferences and datum from past research as the input parameter to prescribe the design of the training programs (Maity, 2019). Relevant research findings and data from the organization are fed into the limited memory of AI, enabling the system to design training programs, including delivery methods and schedules. 


\section{Knowledge Base Building}

An important component of KM is knowledge capture and management. Artificial intelligence can help transform personal knowledge into group knowledge, and give knowledge and information to those who should make the best use of it.

To express knowledge in knowledge bases, the knowledge classification and graph are usually built up as a framework. Knowledge methods and ontologies for expressing acquired knowledge are usually created in the field of artificial intelligence for constructing relative intelligent systems. The KM field can employ artificial intelligence techniques to facilitate the coding of knowledge in KM systems (Liebowitz, 2001).

Knowledge mapping, a series of different graphs showing the process of knowledge development and structural relationships, describes the carrier and knowledge resource using visualization techniques (Wu, 2017). Mesh knowledge mapping constructs structured knowledge system content based on two dimensions, which are then extended to mesh knowledge institutions. The mesh knowledge mapping technology enables the integration and linking of all information in the enterprise knowledge to form an underlying map.

The underlying map is like a very "smart web" that covers all enterprise knowledge and records the connections between knowledge and knowledge (Wu, 2017). When trainees learn online, they can easily access other related learning content directly through links within the site, which greatly increases the depth, breadth, and length of learning. They can learn in-depth along with their expertise, as well as learn all types of related knowledge and even be able to apply it properly through what they have learned.

In addition, to mapping corporate knowledge, the same technology can also be used to build personal maps of employees, mapping their interests, industries, and levels of learning (Wu, 2017). It can also be used to create domain mapping, which is based on domain big data and all user data. Based on the general trends presented by domain mapping and the individual learning level presented by personal mapping, it can intelligently develop a learning path for each learner, helping them to target their learning goals more accurately.

In addition, data mining and knowledge discovery methods can be applied to identify the relationship and find the trend to create new well-structured knowledge systems. It is possible to use knowledge discovery and AI-driven data mining to collect individual knowledge and proper summaries, from the knowledge base and to find trends and relationships between them. This helps to combine existing knowledge, which will mostly cause the generation of new knowledge (Liebowitz, 2001).

Other AI knowledge discovery techniques, such as intelligent agents (Metaxiotis et al., 2004) can be applied to assist with edge search for KM systems. The intelligent agent collects clusters of knowledge from different places and discovers connections between them, thus creating new knowledge for the organization. It exhibits the properties of a reactive mechanical system. For example, it can be used to collect external knowledge, emails, web pages, and online communities, as well as to analyze them (Liebowitz, 2001).

As the computational power of computers increases, machines can "think" on their own without much direct human intervention. 


\section{Training Demand Mining}

Based on the requirements of the position, AI can form customized employee courses through big data analysis for employees who learn from a huge knowledge base. AI can systematically analyze elements such as employees' starting skills, cognitive level, job requirements, and company characteristics, and continuously learn from employees' behavioral data to refine employee portraits to construct unique learning strategies and support employees to learn efficiently.

Based on the analysis of the survey data of trainees, AI can test and position employees' levels comprehensively in technical ways and smartly promote bespoke courses. Learners only input their learning points, profile, and learning objectives and the course will be completed automatically. Natural language processing may rapidly analyze many learner studies. Learners are completely free to write down their ideas in a more free-flowing text format, without even being too logical or chaptered. All textual content is processed by technology in such a way that the core ideas can be distilled completely. This may assist enterprises to better know the needs of their employees and to customize appropriate courses.

Even more, AI can predict the problems employees will encounter according to their personal behavior, the colleagues' behavior, and business requirements, and then provide the appropriate knowledge directly to them at the right moment, achieving the goal of zero-time knowledge transfer.

Furthermore, when organizational leaders diversify the input of skills identification, the skills readiness of employees increases. External markets provide different sources of data that HR departments can use to illuminate around changing skills demands (Baker, 2019). Big data, both internal and external, supplies the input needed to teach the algorithm behind artificial intelligence solutions.

Thus, AI-rich learning systems can provide customizable, individual employee-based training programs. These could potentially contribute to huge increases in workforce productivity, as these AI learning systems could act as personal mentors, providing training to employees, and helping to develop their potential and capabilities (Iqbal, 2018).

\section{Organizing Training}

AI personal training assistants can focus on the learning needs of learners, assess, and diagnose problems and provide assistance during organizational training. It can automatically offer the benefit of one-to-one training, perform tasks to practice their skills in highly interactive learning environments, evaluate trainee behavior, and form a model of their skills, knowledge, and expertise to tailor their teaching strategies, including content and style (Ong \& Ramachandran, 2003). This is demonstrated by the following.

\section{Providing Personalized Trainers}

In one-to-one face-to-face training programs, trainees perform poorly in mastering directed problem solving if they are trained by a human instructor who lacks expressive and organizational skills (Towler \& Dipboye, 2001). For a specific set 
of trainees, AI (a limited memory system) has the potential to input trainer characteristics, migration climate, and research findings to predict the efficiency of training transfer, thus prescribing an AI-based trainer profile (Burke \& Hutchins, 2008).

\section{Meeting Personalized Training Needs}

Artificial intelligence responds to the needs of employees through systems such as adaptive learning programs, games, and software, and collects learning data from employees throughout the process, through which it can analyze not only the macro situation of training but also the micro situation of individual employees' learning habits, learning outcomes, etc., for adjusting employees' training behavior and individualizing training (Zhi, 2019). For example, AI recommends courses that match their "tastes" based on their learning behavior records; automatically knowledge point marker technology marks important knowledge points in the course in the progress bar; by retrieving learning history data, AI analyzes the suitability of the course and recommends a "precursor course" if it is not yet sufficiently knowledgeable; it reduces unnecessary learning and delivers a more concise course, taking into account the level and knowledge base of the trainee.

\section{Micro-dose Learning}

Carmen Fontana, IEEE member and head of cloud computing and emerging technologies at Centric Consulting, calls the new approach to AI-based learning "microdosing" (Korolov, 2021). People do not usually like to spend $40 \mathrm{~h}$ a week in a classroom learning. With AI, this can be done in a very short period with small snippets and tips-so it is timely and easy to absorb. People can get help with these microdoses of learning rather than having formal training. Artificial intelligence can take large chunks of knowledge content, such as video, audio, images, and text, that were originally available to enterprises and automatically break them down into knowledge points that are specific to business issues. These knowledge units and knowledge points form micro-knowledge that can effectively help employees solve specific problems as well as make learning easier. Micro-knowledge makes the learning experience more attractive and flexible with small chunks of learning content, and at the same time greatly reduces the cognitive difficulty and learning pressure of learners, so that employees do not need to spend large chunks of time that could be spent on leisure to learn easily. The micro-dose learning approach, however, may be divorced from the original overall context. This runs the risk of misleading information. Therefore, when adopting such a learning approach, it should also be conscious of the fact that knowledge is usually part of a "larger" context, but using only part of it can quickly degrade its quality. 


\section{Robotic Q\&A}

Robotics and autonomous agent chatbot applications are a major trend in the future of AI-based training and fall under the real-time nature of artificial intelligence (Krithika et al., 2019). Many enterprises have already incorporated chatbots into their HR training systems to provide answers to employee queries. The human-computer interaction of AI enables timely answers to employees' queries and enables them to learn anytime, anywhere. The "smart quiz" is based on semantic parsing of questions and keyword matching to give quick answers to standard statements. Trainees can upload their questions to the training system with the help of artificial intelligence voice recognition and image recognition technologies, and then analyze and integrate the questions with the central database of the training system to obtain the answers. Questions that cannot be solved by "smart answers" can be answered by AI inviting experts or colleagues from the enterprise to answer them, and the Q\&A content will be deposited in the enterprise's knowledge base for later access (Celia, 2019). The question and answer are fed into a central database and the machine can train to be smarter. As trainees use it more, the more questions they can answer accurately.

\section{Observing a Learning State}

During training, the robot trainer can observe the usual learning performance of the trainees through a visual feedback lidar scanning system, precisely calculate the average value of everyone's attention, and then use data analysis to extract the teaching events at various stimulus levels. It is also possible to adjust the pace and relaxation of the teaching based on the feedback from the participants (Oshima et al., 2012). The platform also records the learning data of students, such as learning hotspots time, frequency abundance, duration, content preferences, and user outcome data (e.g., learning efficiency, test scores, practice scoring), which is data-matched with People Analytics to interpret the learning behavior of the employees through the analysis of each dimension for further growth analysis.

\section{Scenario Simulation}

Traditional teaching methods inculcate "inert knowledge" that trainees can recall, but cannot apply flexibly in practice. AI technology may help people to apply what they have learned by the function of learning environment simulations. These flexible, contextualized teaching and learning environments help to apply and retain skills and knowledge. Contemporary, more welcoming virtual reality (VR) and augmented reality (AR) technologies can simulate realistic scenarios for trainees, making learning more immersive and engaging for users, and enhancing the usefulness and practicality of training.

In short, AI-based training and learning can help personalize the learning experience. Learning content is intelligently recommended and assigned to everyone according to past completion, interaction with various types of learning content, present skill demands, and future career development (Baker, 2019). 


\section{Training Outcomes Response}

Ali et al. (2015) point out the potential of AI and the computing system to support not only training needs identification and educational action planning, but also the assessment of training outcomes.

The results of training are not only related to the performance of the company and the performance of the trainees at work (e.g., personal self-efficacy and selfmanagement) but also to the well-being of the employees (e.g., problem-solving skills, planning, communication, improvement of shared task development).

AI teachers can be assistants in all aspects of training, from analyzing the learning data to the output of learning reports and from the supervision of trainees to the automatic ranking of learning, helping trainees to automatically record training data, assess everyone's performance, and provide them with useful comments and feedback during the training process. Visual data is also analyzed to express the extent and effectiveness of trainee learning, allowing the organization to quickly understand the results of the training.

Artificial intelligence systems can make pay recommendations to managers based on training results to encourage employees to improve their skills. IBM, for example, correlates employee pay with skill sets and addresses pay inequality (Korolov, 2021).

Furthermore, AI can detect gaps in the learning product by monitoring the results of employees' learning, leading to more focused improvements in format and content (Baker, 2019).

\section{Management Challenges}

This study proposes management challenges and corresponding responses that organizations should be aware of.

\section{Operational Costs and Database}

According to Kaizer et al. (2020), workplace training is a core element in strengthening innovative enterprises. Through educational initiatives, workers are a source of profit creation rather than a "cost." Therefore, proper training investment is beneficial for the development of employees and the organization.

However, developing a good AI tool requires good algorithms, which is expensive, so every organization may not be able to afford so much technology and the cost of rapidly growing complex algorithms (Saju Mathew et al., 2021). The high cost of investment and uncertain ROI are the primary reasons that prevent organizations from using the AI tool.

Most organizations have a limited number of employees and will have a limited dataset. Depending on the teaching algorithm explained, organizations may have a limited number of trainee profiles stored, so it is difficult to teach algorithms or 
AI tools to perform according to human resources (HR) and organizational requirements. Sometimes AI tools may not correctly interpret the results of misinformation (Saju Mathew et al., 2021).

To maintain a competitive advantage, organizations can develop strategic plans for AI-based training transformation by gradually constructing internal data analysis systems and improving information systems. Doing so will reduce cost pressures and will also provide a database for embedding AI systems.

\section{Data Security and Legal Risks}

AI-based training solutions can add value and people take a pragmatic approach to data protection and privacy (Lichtenthaler, 2019). It is considered that one of the important factors in developing a good AI tool depends on the availability of data and the security of the resources they need (Saju Mathew et al., 2021). Beyond the advantages that AI-based training brings to human resources, there may be risks like data leakage and unintentional misuse (Josh, 2018). To develop AI for training decisions, a large amount of data needs to be collected from numerous sources. This data can come from internal or external sources, or both (Akerkar, 2019). Extracting additional internal information may lead to legal, ethical, and privacy issues. In addition, the use of external datasets may pose some legal privacy challenges (Chichester Jr \& Giffen, 2019).

It is recommended that AI-based training system developers build data security and privacy prevention tools as well as usage policies. In addition, they need to be aware of the legal requirements regarding access to data. However, once laws are passed or people start to significantly restrict their access to data from external sources, this could seriously affect the efficiency of AI promotion tools.

\section{Job Replacement}

Artificial intelligence processes may take over repetitive tasks previously performed by humans, leading to the redundancy of certain positions (e.g., trainers) (Iqbal, 2018). Such replacement has also caused considerable public attention, with speculation regarding many jobs that could be lost in such a shift (Winick, 2018). The rise in unemployment can also lead to opposition and resistance. How to apply the developing technologies to achieve human-machine collaboration is also an important challenge.

However, the process of communicating with people requires emotional transfer, emotional transformation, and communication skills. This alone is one of the core skills that HR workers have developed over the years and will not be easily replaced by artificial intelligence.

Furthermore, the emergence of AI in training will eliminate tedious and repetitive jobs such as interpreting needs evaluation investigation, organizing training plans, delivering, and selecting trainers, and measuring the organizational learning climate and working environment. These tedious transactional tasks of training can be left to artificial intelligence. Thus, by integrating AI with training, it helps people to focus more on strategic tasks rather than repetitive tasks with low added value. It provides 
a new dimension to human resources (HR) to streamline and reshape it so that it can achieve better productivity and help reduce costs and automate repetitive tasks (Saju Mathew et al., 2021). Soon, the role of the human training designer will become more strategic and decision-making, such as developing training practice strategies, facilitating, and monitoring training transfer and innovating training methods.

\section{Equity}

Artificial intelligence ethics is a management issue (Siau \& Wang, 2020). Managers must remain legally and ethically responsible when using AI in decision-making. The technology may contain intentional or unintentional bias, as well as a lack of human intelligence, emotions, and values.

One of the advantages of an artificial intelligence tool is that it does not show bias or reduce the bias caused by human interference. However, if the system is not programmed correctly, then it will show some sort of bias (Saju Mathew et al., 2021).

Continuous human control and intervention can be used to minimize the risks associated with transitioning to an AI system in an organization. This tool needs to be trained on data, due to the fact that the results are based on the analysis and recognition of data patterns (Saju Mathew et al., 2021).

\section{Negative Attitudes}

It is difficult for people to predict how an AI tool will behave with input in a given situation (Saju Mathew et al., 2021). Some people are more comfortable working with a real person than a virtual colleague without human interaction. Negative employee attitudes may emerge due to the ambiguity related to the unstable human-machine interface. Drawing on previous research on newly invented attitudes (Lichtenthaler, 2019), the attitude is referred to as a no-human-interaction (NHI) attitude, and it is defined as the negative attitude towards interacting with artificial intelligence (Lichtenthaler, 2019).

As business competition increases, the use of AI in business becomes more prevalent. Organizations shape a climate culture that is inclusive and accepting of new things, advancing the idea that employees can embrace new things with a more tolerant attitude. The benefits of training brought by AI can make people aware that AI can take away tedious tasks for us. Some operations or regions could try AI-based training, and once the trial is successful, it could be rolled out across the organization.

Other causes of negative attitudes about AI among some employees may be retraced to employees' apprehension of losing their jobs due to the potential for technology to replace human work (Winick, 2018).

This replacement of human jobs represents only a small portion of the possible competitive advantage, as in the long run, the synergy between artificial intelligence and 
human intelligence will provide more significant benefits than simply replacing human jobs (Lichtenthaler, 2018).

\section{Training Effectiveness Measurement and Feedback}

Most organizations find it difficult to measure the effectiveness of training on an irregular basis (Srimannarayana, 2011). They consider the limitations of existing measurement models and the lack of control by training professionals over the data needed to measure training and development.

Organizations can identify and define relevant parameters and use existing measurement models or develop new ones to measure the ROI of the training programs undertaken and their impact on the business. This can then be used as recursive analysis and feedback to improve the organization's training and development activities (Maity, 2019).

\section{Result of the Research}

This study shows that as technology advances, more and more organizations are turning to AI-based training. The traditional classroom model is not well adapted to personalized teaching and learning. AI-based training allows personalization needs to be greatly met. In specific training practices, AI tools can be involved in knowledge base building, training demand mining, organization training, and result feedback. Especially in the organizational training stage, AI tools can provide personalized trainers, personalized demand satisfaction, micro-dose learning, learning status tracking, robot Q\&A, and scenario simulation. The participation of AI technology greatly improves the training effect, reduces the workload of enterprise trainers, and makes personalized training demand satisfied. However, AI-based training also raises a number of challenges, such as high technology costs, data privacy issues, HR staff job replacement, training equity, and how training results are evaluated. While AI tools bring revolutionary changes to organizations' training practices, these challenges cannot be ignored. It is possible that the impact of these challenges will reduce or even offset the benefits of AI-based training. In future training practices as well as research, it is important to consider how to better leverage AI-based training and be able to address the negative issues it brings.

\section{Limitations and Conclusions}

Future AI-based training research could consider quantitative analysis and quantitative cross-sectional studies with reference to different countries, cultures, and metrics across companies and gender.

Current training has transitioned from a traditional teaching model to a flexible electronic and AI system-supported approach. AI-based training transforms 
organizations into knowledge organizations that can meet the demands of personalized training and improve learning quality. AI tools bring about a shift in the training phases of knowledge base creation, needs survey, the organization of training, and feedback on results. The costs, privacy, job substitutability, fairness, negative attitudes, and feedback on the training outcomes also need to be given high priority by the organization. AI-based training is just emerging and has research limitations, but it also gives the opportunity for more exploration.

Acknowledgements The authors would like to acknowledge the project team and the collaboration effort of the wider project team, which included Professor Sun Jie and Ms. Han Ying.

\section{Declarations}

Ethics Statement No animals or humans were involved in the study.

Conflict of Interest The author declares no competing interests.

\section{References}

Abbad, G. D. S., \& Borges-Andrade, J. E. (2004). Aprendizagem humana em organizações de trabalho. Psicologia, organizações e trabalho no Brasil, 2, 237-275.

Aguinis, H., \& Kraiger, K. (2009). Benefits of training and development for individuals and teams, organizations, and society. Annual review of psychology, 60, 451-474.

Akerkar, R. (2019). Artificial intelligence for business. Springer.

Ali, M., Kwon, Y. S., Lee, C. -H., Kim, J., \& Kim, Y. (2015). Current approaches in applied artificial intelligence: 28th International Conference on Industrial, Engineering and Other Applications of Applied Intelligent Systems, IEA/AIE 2015, Seoul, South Korea, June 10-12, 2015, Proceedings (Vol. 9101). Springer.

Baker, M. (2019). Leverage Artificial Intelligence in HR Processes Where it Matters Most. Available at: https://www.gartner.com/smarterwithgartner/leverage-artificial-intelligence-in-hr-processes-whereit-matters-most.

Bell, B. S., Tannenbaum, S. I., Ford, J. K., Noe, R. A., \& Kraiger, K. (2017). 100 years of training and development research: What we know and where we should go. Journal of Applied Psychology, $102(3), 305$.

Bray, A., \& Tangney, B. (2016). Enhancing student engagement through the affordances of mobile technology: A 21st century learning perspective on Realistic Mathematics Education. Mathematics Education Research Journal, 28(1), 173-197.

Burke, L. A., \& Hutchins, H. (2008). A study of best practices in training transfer and proposed model of transfer. Human resource development quarterly, 19(2), 107-128.

Carayannis, E. G., Barth, T. D., \& Campbell, D. F. J. (2012). The Quintuple Helix innovation model: Global warming as a challenge and driver for innovation. Journal of Innovation and Entrepreneurship, 1(1), 2. https://doi.org/10.1186/2192-5372-1-2

Carayannis, E. G., \& Campbell, D. F. J. (2014). Developed democracies versus emerging autocracies: Arts, democracy, and innovation in Quadruple Helix innovation systems. Journal of Innovation and Entrepreneurship, 3(1), 12. https://doi.org/10.1186/s13731-014-0012-2

Carayannis, E. G., \& Campbell, D. F. J. (2021). Democracy of climate and climate for democracy: The evolution of Quadruple and Quintuple Helix innovation systems. Journal of the Knowledge Economy, 12(4), 2050-2082. https://doi.org/10.1007/s13132-021-00778-x

Carayannis, E. G., Campbell, D. F. J., \& Grigoroudis, E. (2021). Helix Trilogy: The Triple, Quadruple, and Quintuple Innovation Helices from a theory, policy, and practice set of perspectives. Journal of the Knowledge Economy. https://doi.org/10.1007/s13132-021-00813-x 
Celia. (2019). AI energizes training from three sides. Available at: http://www.ruthout.com/information/ 19806.html

Chichester, M. A., Jr., \& Giffen, J. R. J. C. I. L. (2019). Recruiting in the Robot Age: Examining Potential EEO Implications in Optimizing Recruiting through the Use of Artificial Intelligence., 36(10), 1-3.

Ellinger, A. D., \& Cseh, M. (2007). Contextual factors influencing the facilitation of others' learning through everyday work experiences. Journal of Workplace learning.

Ellis, A. P., Bell, B. S., Ployhart, R. E., Hollenbeck, J. R., \& Ilgen, D. R. (2005). An evaluation of generic teamwork skills training with action teams: Effects on cognitive and skill-based outcomes. Personnel psychology, 58(3), 641-672.

Haenlein, M., \& Kaplan, A. (2019). A brief history of artificial intelligence: On the past, present, and future of artificial intelligence. California management review, 61(4), 5-14.

Iqbal, F. M. (2018). Can artificial intelligence change the way in which companies recruit, train, develop and manage human resources in workplace?. Asian Journal of Social Sciences and Management Studies, 5(3), 102-104.

Jia, Q., Guo, Y., Li, R., Li, Y., \& Chen, Y. (2018). A conceptual artificial intelligence application framework in human resource management. Proceedings of the International Conference on Electronic Business.

Josh, B. (2018). AI in HR: a real killer App. In. Available at: https://www.forbes.com/sites/joshbersin/ 2018/06/18/ai-in-hr-a-real-killer-app/\#59637a8348f1

Kaizer, B. M., Silva, C. E. S., de Pavia, A. P., \& Zerbini, T. (2020). E-learning training in work corporations: A review on instructional planning. European Journal of Training and Development.

Korolov, M. (2021). How does artificial intelligence completedly reform employee' training?. Available at: https://ai.51cto.com/art/202104/659543.htm

Krithika, J., Venkatraman, P., \& Sindhujaa, E. (2019). Virtual HR era in human resource management. EPRA International Journal of Multidisciplinary Research, 5(10), 49-57.

Lichtenthaler, U. (2019). Extremes of acceptance: employee attitudes toward artificial intelligence. Journal of Business Strategy.

Lichtenthaler, U. (2018). Substitute or synthesis: the interplay between human and artificial intelligence. Research-technology management, 61(5), 12-14.

Liebowitz, J. (2001). Knowledge management and its link to artificial intelligence. Expert systems with applications, 20(1), 1-6.

LiT, J., Wang, T., \& Yuan, B. (2019). New module research of enterprises' training and development based on AI technology. Modern Shopingmall, 000(008), 75-76. CNKI:SUN:SCXH.0.2019-08-044

Luckin, R., \& Cukurova, M. (2019). Designing educational technologies in the age of AI: A learning sciences-driven approach. British Journal of Educational Technology, 50(6), 2824-2838.

Maity, S. (2019). Identifying opportunities for artificial intelligence in the evolution of training and development practices. Journal of Management Development.

Metaxiotis, K., Ergazakis, K., Samouilidis, E., \& Psarras, J. (2004). Decision support through knowledge management: the role of the artificial intelligence. International Journal of Computer Applications in Technology, 19(2), 101-106.

Noe, R. A., Clarke, A. D., \& Klein, H. J. J. A. R. O. P. O. B. (2014). Learning in the Twenty-First-Century Workplace., 1(1), 245-275.

Ong, J., \& Ramachandran, S. J. N. N. (2003). Intelligent Tutoring Systems: Using Ai to Improve Training Performance and Roi, 19(6), 1-6.

Oshima, J., Oshima, R., \& Miyake, N. (2012). Collaborative reading comprehension with communication robots as learning partners. Proceedings of International Conference of the Learning Sciences (pp.). ICLS, Sydney, Australia, July, pp. 256-267.

Poll, A. E., Zyl, I. V., \& Kroeze, J. H. (2021). Social exclusion in gamified information systems. Conference on e-Business, e-Services and e-Society.

Raab, R. T., Ellis, W. W., \& Abdon, B. R. (2001). Multisectoral partnerships in e-learning: A potential force for improved human capital development in the Asia Pacific. The Internet and higher education, 4(3-4), 217-229.

Reiser, R. A. (2001). A history of instructional design and technology: Part II: A history of instructional design. Educational technology research and development, 49(2), 57-67.

Rossett, A. (2009). First things fast: A handbook for performance analysis. John Wiley \& Sons.

Saju Mathew, D., Oswal, N., \& Ateeq, K. (2021). Artificial intelligence (AI): Bringing a new revolution in human resource management (HRM). 
Salas, E., DiazGranados, D., Klein, C., Burke, C. S., Stagl, K. C., Goodwin, G. F., \& Halpin, S. M. (2008). Does team training improve team performance? A Meta-Analysis. Human factors, 50(6), 903-933.

Salas, E., Tannenbaum, S. I., Kraiger, K., \& Smith-Jentsch, K. A. (2012). The science of training and development in organizations: What matters in practice. Psychological science in the public interest, 13(2), 74-101.

Siau, K., \& Wang, W. (2020). Artificial intelligence (AI) ethics: ethics of AI and ethical AI. Journal of Database Management (JDM), 31(2), 74-87.

Srimannarayana, M. (2011). Measuring training \& development. Indian Journal of Industrial Relations, $117-125$.

Taylor, K. (2017). The future of corporate learning: Stepping into another dimension. Training industry magazine, 10(4), 34-39.

Torraco, R. J. (2016). Early history of the fields of practice of training and development and organization development. Advances in Developing Human Resources, 18(4), 439-453.

Towler, A. J., \& Dipboye, R. L. (2001). Effects of trainer expressiveness, organization, and trainee goal orientation on training outcomes. Journal of Applied Psychology, 86(4), 664.

Werner, J. M., \& DeSimone, R. L. R. L. (2011). Human resource development. Cengage Learning.

Winick, E. (2018). Every study we could find on what automation will do to jobs, in one chart. Technology Review.

Wu, H. (2017). AI-based training application in Baidu Corporation. Available at: http://news.youth.cn/ gn/201711/t20171101_10949452.htm

Zahra, S., Iram, A., \& Naeem, H. (2014). Employee training and its effect on employees' job motivation and commitment: Developing and proposing a conceptual model. IOSR Journal of Business and Management, 16(9), 60-68.

Zhi, X. (2019). Look at how AI cooperates with training in future. Available at: https://baijiahao.baidu. $\mathrm{com} / \mathrm{s} ? \mathrm{id}=1638477710576603915 \& \mathrm{wfr}=$ spider $\&$ for $=\mathrm{pc}$

Publisher's Note Springer Nature remains neutral with regard to jurisdictional claims in published maps and institutional affiliations. 